\title{
MicroRNA Expression in the Aqueous Humor of Patients with Diabetic Macular Edema
}

\author{
Giuseppina Emanuela Grieco ${ }^{1,2} \mathbb{D}^{0}$, Guido Sebastiani ${ }^{1,2}$, Chiara Maria Eandi ${ }^{3,4}$, Giovanni Neri ${ }^{5}$, \\ Laura Nigi ${ }^{1,2}$, Noemi Brusco ${ }^{1,2}$, Romina $D^{\prime}$ Aurizio ${ }^{6}{ }^{(}$, Matteo Posarelli ${ }^{5}$, Tommaso Bacci ${ }^{5}$, \\ Elena De Benedetto ${ }^{5}$, Mario Fruschelli ${ }^{5}$, Maurizio Orlandini ${ }^{7}$, Federico Galvagni ${ }^{7}{ }^{\mathbb{D}}$, \\ Francesco Dotta ${ }^{1,2, *}$ and Gian Marco Tosi ${ }^{5, *}$ \\ 1 Diabetes Unit, Department of Medicine, Surgery and Neurosciences, University of Siena, Viale Bracci, 16, \\ 53100 Siena, Italy; giusy.grieco.90@gmail.com (G.E.G.); sebastianiguido@gmail.com (G.S.); \\ launigi@gmail.com (L.N.); noemibrusco91@gmail.com (N.B.) \\ 2 Fondazione Umberto Di Mario ONLUS c/o Toscana Life Sciences, 53100 Siena, Italy \\ 3 Department of Surgical Science, University of Torino, 10124 Torino, Italy; chiara.eandi@unito.it \\ 4 Department of Ophthalmology, University of Lausanne, Jules-Gonin Eye Hospital, \\ 1004 Lausanne, Switzerland \\ 5 Ophthalmology Unit of the Department of Medicine, Surgery and Neuroscience, University of Siena, \\ 53100 Siena, Italy; gio.neri2009@libero.it (G.N.); mposarelli@gmail.com (M.P.); \\ osammot.iccab@gmail.com (T.B.); elenadebenedetto1992@gmail.com (E.D.B.); mario.fruschelli@unisi.it (M.F.) \\ 6 Institute of Informatics and Telematics, National Research Council, 56124 Pisa, Italy; \\ romina.daurizio@gmail.com \\ 7 Department of Biotechnology, Chemistry and Pharmacy, University of Siena, 53100 Siena, Italy; \\ maurizio.orlandini@unisi.it (M.O.); federico.galvagni@unisi.it (F.G.) \\ * Correspondence: francesco.dotta@unisi.it (F.D.); gianmarco.tosi@unisi.it (G.M.T.)
}

Received: 8 September 2020; Accepted: 30 September 2020; Published: 3 October 2020

\begin{abstract}
We identified and compared secreted microRNA (miRNA) expression in aqueous humor (AH) and plasma samples among patients with: type 2 diabetes mellitus (T2D) complicated by non-proliferative diabetic retinopathy (DR) associated with diabetic macular edema (DME) (DME group: 12 patients); T2D patients without DR (D group: 8 patients); and non-diabetic patients (CTR group: 10 patients). Individual patient AH samples from five subjects in each group were profiled on TaqMan Low Density MicroRNA Array Cards. Differentially expressed miRNAs identified from profiling were then validated in single assay for all subjects. The miRNAs validated in AH were then evaluated in single assay in plasma. Gene Ontology (GO) analysis was conducted. From AH profiling, 119 mature miRNAs were detected: 86 in the DME group, 113 in the D group and 107 in the CTR group. miRNA underexpression in the DME group was confirmed in single assay for let-7c-5p, miR-200b-3p, miR-199a-3p and miR-365-3p. Of these four, miR-199a-3p and miR-365-3p were downregulated also in the plasma of the DME group. GO highlighted 54 validated target genes of miR-199a-3p, miR-200b-3p and miR-365-3p potentially implied in DME pathogenesis. Although more studies are needed, miR-200b-3p, let-7c-5p, miR-365-3p and miR-199a-3p represent interesting molecules in the study of DME pathogenesis.
\end{abstract}

Keywords: type 2 diabetes mellitus; diabetic retinopathy; Diabetic Macular Edema; microRNA; miRNA; aqueous humor

\section{Introduction}

Diabetic retinopathy (DR) represents a highly specific vascular complication of type 1 (T1D) and type 2 (T2D) diabetes and is the leading cause of visual impairment in working-age adults [1]. 
Chronic hyperglycemia activates different pathways, involving multiple cytokines, eventually leading to the dysfunction of the retinal neuronal-glial-vascular unit [2]. Vascular endothelial growth factor A (VEGFA) is the most studied cytokine implicated in DR pathogenesis. However, growing evidence shows that the processes triggered by chronic hyperglycemia are more complex and may not be driven by VEGFA alone [2-6].

As a matter of fact, not only protein-coding genes but also noncoding RNAs (ncRNAs) are involved in DR molecular signaling interplay, thus rendering understanding of this condition even more complex [7,8].

MicroRNAs (miRNAs) are short (about 22 nucleotides long) and highly conserved sequences of endogenous ncRNAs that represent a powerful class of gene modulators involved in the main biological processes, including cell growth, differentiation and apoptosis. MiRNAs have very long half-lives and have been found to be stable in many biological fluids, including human serum, plasma, urine, saliva, tears, aqueous humor $(\mathrm{AH})$ and vitreous humor $[7,9]$.

With the aim of identifying new biomarkers and possible additional therapeutic targets, numerous miRNAs have been studied in DR, but this has mainly been performed using cell lines and/or animal models, and only rarely on human samples [10]. In fact, human plasma/serum sampling has only been performed in a few case-control studies recruiting either T1D or T2D patients [11-15]. Investigations using human intraocular samples (vitreous and aqueous humor) are even rarer. Gomaa et al. [16] sampled the vitreous, evaluating miR-200b expression in both T1D and T2D patients, while Usui-Ouchi et al. [17], Hirota et al. [18] and Mammadzada et al. [19] evaluated the expression of multiple miRNAs through a microarray or PCR array vitreal profile. Recently, Chen et al. [20] performed next generation sequencing to evaluate multiple miRNA expression in AH of T2D patients. In the above-mentioned vitreal studies and in the aqueous study, patients recruited were affected by proliferative diabetic retinopathy (PDR). However, based on results in human serum $[11,13]$ and in animal models of diabetic retinopathy [2-7], different stages of DR might be associated with different expression of both miRNAs and cytokines, leading researchers to propose a "liquid biopsy" immediately prior to the first intraocular injection in order to identify the predominant pathogenic pathway in a patient-specific manner [21]. To our knowledge, only Cho and colleagues [22] evaluated miRNA expression in intraocular samples of patients suffering from non-proliferative diabetic retinopathy (NPDR) complicated by diabetic macular edema (DME). This study was performed by a PCR array composed of 84 miRNAs, chosen for their association with inflammation and angiogenesis, and conducted using cataract patients as control subjects. However, an unbiased miRNAs profiling in a similar population has not been performed yet.

In order to increase the knowledge of this stage of DR, we performed a TaqMan array profiling of 378 miRNAs in the aqueous humor of patients with T2D-associated NPDR complicated by DME; in T2D patients without DR; and in patients without diabetes. In addition, differentially expressed microRNAs of interest were analyzed in plasma samples from the same patients in order to identify miRNAs, whose expression in plasma could mirror that occurring in aqueous humor.

\section{Results}

The baseline characteristics of the patient groups studied are summarized in Table 1. No between-groups differences were present for age at sampling, Body Mass Index (BMI), or comorbidities.

miRNA expression profiling was performed in AH samples using TaqMan miRNA arrays, which allowed the evaluation of 378 miRNA expression levels in $n=5$ patients per group. Each miRNA analyzed was considered detected when resulting cycle threshold $(\mathrm{Ct})$ was $\leq 35.0$ in at least 4 out of 5 subjects in at least one group. Overall, based on these criteria, a total of 119 miRNAs were reliably detected in AH samples (Figure 1a and Supplementary File S1). 
Table 1. Clinical characteristics of patients.

\begin{tabular}{|c|c|c|c|}
\hline Characteristics & CTR & D & DME \\
\hline Demographic, $n=$ & 10 & 8 & 12 \\
\hline Age, mean (SD; range), years & $71(4.8 ; 65-78)$ & $72(5,1 ; 65-78)$ & $69(4.9 ; 57-79)$ \\
\hline \multicolumn{4}{|c|}{ Sex, No. (\%) } \\
\hline Male & $7(70 \%)$ & $6(75 \%)$ & $8(67 \%)$ \\
\hline Female & $3(30 \%)$ & $2(33 \%)$ & $4(33 \%)$ \\
\hline BMI, mean (SD; range) & $25(3.2 ; 22-33)$ & $28(2.6,26-34)$ & $27(1.8 ; 23-30)$ \\
\hline \multicolumn{4}{|c|}{ Comorbidities, No. (\%) } \\
\hline No comorbidities & $2(20 \%)$ & - & - \\
\hline HTN & $4(40 \%)$ & $8(100 \%)$ & $12(100 \%)$ \\
\hline $\mathrm{BPH}$ & $2(20 \%)$ & $3(37 \%)$ & - \\
\hline MI & $1(10 \%)$ & - & $2(17 \%)$ \\
\hline DLP & $2(20 \%)$ & $2(25 \%)$ & $3(25 \%)$ \\
\hline Others & $2(20 \%)$ & $2(25 \%)$ & $2(17 \%)$ \\
\hline \multicolumn{4}{|c|}{ Treatment, No. (\%) } \\
\hline Metformin & - & $5(62 \%)$ & $5(42 \%)$ \\
\hline Insulin & - & $2(25 \%)$ & $7(58 \%)$ \\
\hline Other diabetic drugs & - & $3(37 \%)$ & $3(25 \%)$ \\
\hline HTN drugs & $5(50 \%)$ & $7(87 \%)$ & $11(92 \%)$ \\
\hline Antiplatelet drugs & $1(10 \%)$ & $3(37 \%)$ & $3(25 \%)$ \\
\hline BPH drugs & $2(20 \%)$ & $2(25 \%)$ & - \\
\hline DLP drugs & $2(20 \%)$ & $2(25 \%)$ & $3(25 \%)$ \\
\hline Others & $2(20 \%)$ & $3(37 \%)$ & $3(25 \%)$ \\
\hline \multicolumn{4}{|c|}{ Laterality, No. (\%) } \\
\hline Right & $4(40 \%)$ & $4(50 \%)$ & $5(42 \%)$ \\
\hline Left & $6(60 \%)$ & $4(50 \%)$ & $7(58 \%)$ \\
\hline \multicolumn{4}{|c|}{ Best-corrected Visual Acuity } \\
\hline Snellen, mean (range) & $20 / 40(20 / 25-20 / 50)$ & $20 / 40(20 / 32-20 / 63)$ & $20 / 40(20 / 25-20 / 100)$ \\
\hline LogMAR, mean (SD; range) & $0.3(0.1 ; 0.1-0.4)$ & $0.3(0.1 ; 0.2-0.5)$ & $0.3(0.2 ; 0.1-0.7)$ \\
\hline \multicolumn{4}{|c|}{ OCT Features } \\
\hline $\begin{array}{l}\text { Central subfield thickness, } \\
\text { mean (SD; range), } \mu \mathrm{m}\end{array}$ & $272.4(12.3 ; 251-289)$ & $275(8.2 ; 263-289)$ & $447.7(86.6 ; 319-604)$ \\
\hline \multicolumn{4}{|c|}{ Grading of Diabetic Retinopathy, No. (\%) } \\
\hline Mild & Not applicable & absent & 6 \\
\hline Moderate & Not applicable & absent & 6 \\
\hline Severe & Not applicable & absent & absent \\
\hline $\begin{array}{l}\text { Duration of diabetes, } \\
\text { mean (SD; range), years }\end{array}$ & Not applicable & $7(1.5 ; 5-10)$ & $6(2.7 ; 1-11)$ \\
\hline HbA1c, mean (SD; range) & Not applicable & $7(0.3 ; 6.9-7.8)$ & $8.0(1.23 ; 6.6-10.2)$ \\
\hline
\end{tabular}

In particular, among the 119 detected miRNAs, 83 were commonly identified in all three groups, while 19 resulted commonly detected between CTR and D groups. Furthermore, 4 miRNAs resulted exclusively expressed in CTR patients, 10 miRNAs were identified only in D group and 1 miRNA was exclusively detected in DME group (Figure 1b).

Considering each group separately, 107/378 miRNAs were detected in the CTR group, 113/378 in the D group and 86/378 miRNAs in the DME group (Supplementary Table S2), highlighting a similar output between CTR and D groups (in terms of miRNAs number), and a significant lower number 
of miRNAs detected in DME AH samples respect to both CTR and D (Supplementary Table S2 and Supplementary File S1).

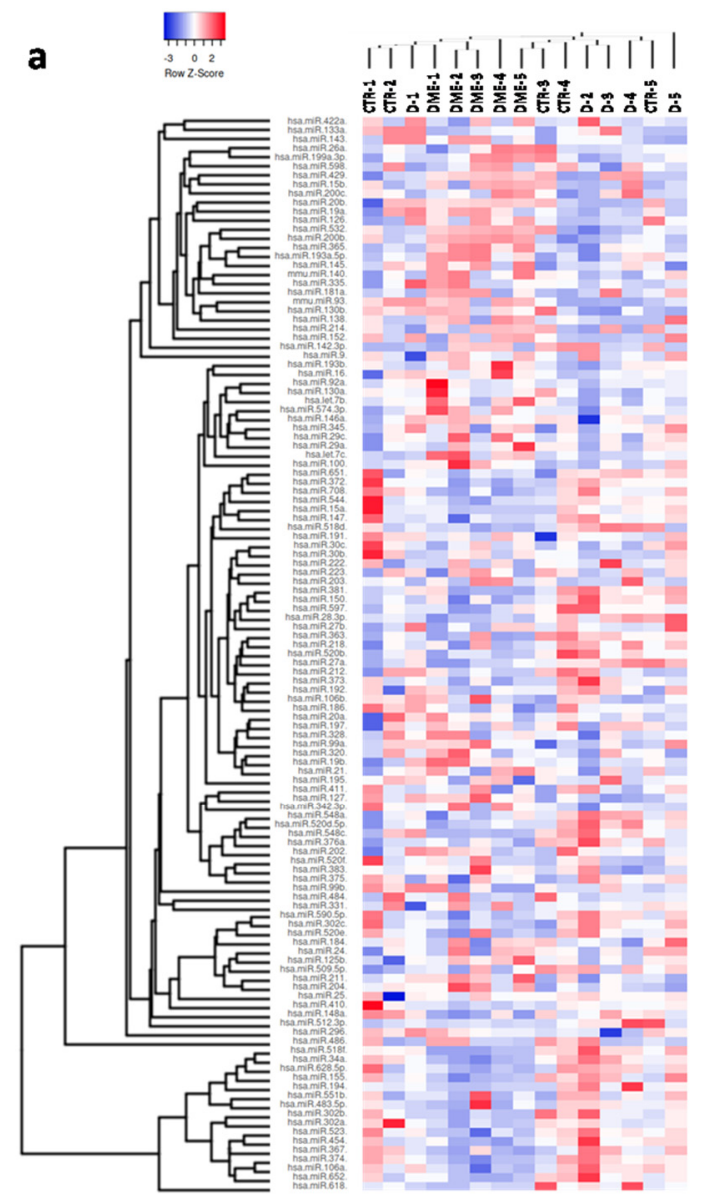

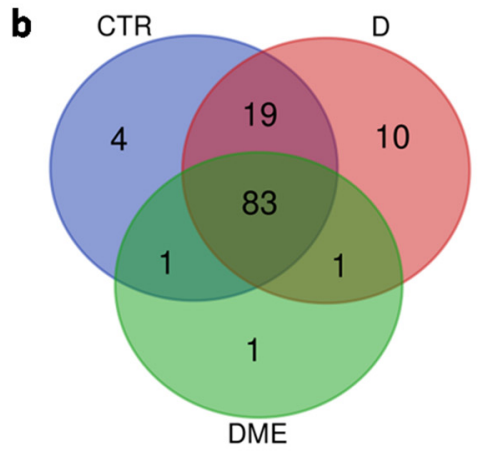

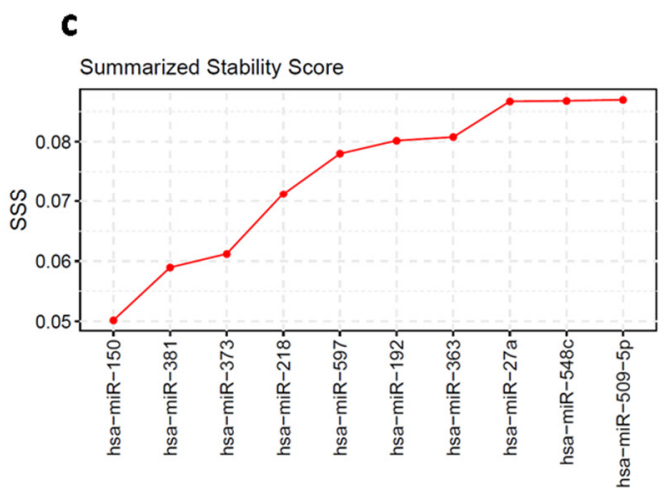

Figure 1. (a) Hierarchical Clustering Heatmap analysis of miRNAs detected $(n=119)$ in at least $4 / 5$ sample for each group. A total of 15 subjects were analyzed: $n=5$ non diabetic controls (CTR), $n=5$ type 2 diabetic patients (D) and $n=5$ type 2 diabetic with Diabetic Macular Edema patients (DME). MicroRNAs expression levels are reported as scale colors based on $\triangle \mathrm{CT}$ expression (Blue, high expression: $\Delta \mathrm{CT}=-3$; Red, low expression: $\triangle C T=2$. (b) Venn diagram showing miRNAs distribution among the three groups; in particular, 83 miRNAs were common among the three groups, while 19 miRNAs were exclusively found in CTR and D but not in DME group; moreover, four miRNAs were exclusively detected in CTR groups and 10 miRNAs resulted exclusively expressed in D group, while only 1 miRNA is exclusively detected in DME group. (c) Stability ranking of top 10 candidate reference miRNAs based on SSS metric which combined three different assessment algorithms. Most stable miRNAs presented lowest SSS values.

In order to compare miRNAs expression levels among the analyzed groups and reduce RT-qPCR data variation, we implemented a normalization step by applying two different strategies: (i) global mean normalization; (ii) best reference miRNAs through combined algorithms normalization. Consequently, the three best-performing miRNAs (miR-150-5p, miR-381-3p and miR-373-3p) (Figure 1c and Supplementary Figure S1a-c) according to SSS metric were chosen to normalize miRNAs expression levels. Therefore, differentially expressed miRNAs were selected from among those identified upon normalization using both strategies.

According to such criteria, 11 miRNAs were differentially expressed between DME and CTR groups ( 3 miRNAs upregulated and 8 downregulated in the DME group) (Figure 2a,d and Table 2) and 15 between the DME and D groups (4 miRNAs upregulated and 11 downregulated in the 
DME group) (Figure $2 \mathrm{~b}, \mathrm{e}$, and Table 2), while 2 miRNAs were differentially expressed between D and CTR groups (miR-548a-3p downregulated and miR-130a-3p upregulated in D group vs. CTR) (Figure 2c,f). Interestingly, among these miRNAs, 8 resulted commonly differentially expressed among the comparisons DME vs CTR and DME vs D (Table 2 and Figure 3).

\section{SSS Normalization}
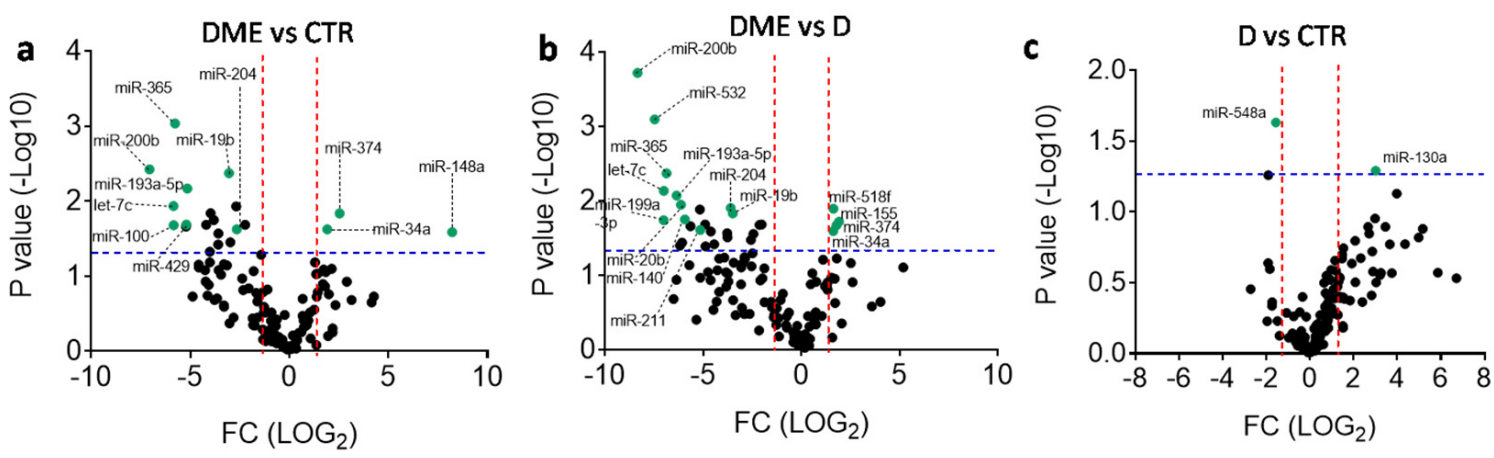

\section{Global Mean Normalization}

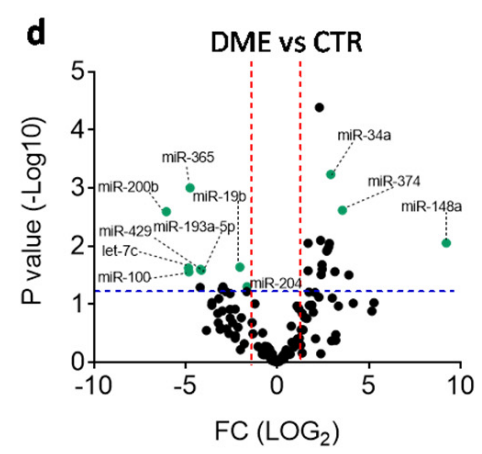

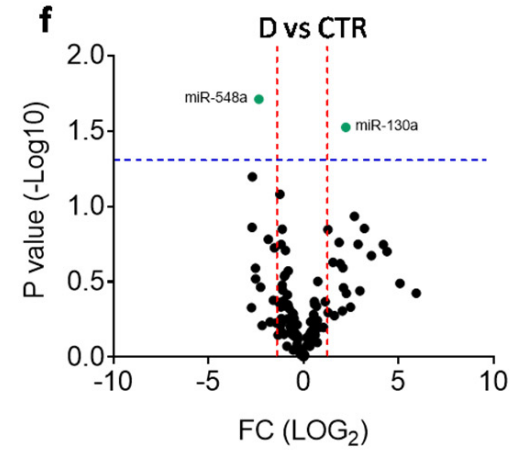

Figure 2. Volcano plots showing differentially expressed miRNAs according to two different normalization strategies. (a-c) Volcano plots of differentially expressed miRNAs in DME vs CTR (a), DME vs. D (b), and D vs. CTR patient groups (c) computed using SSS normalization strategy. $(\mathbf{d}-\mathbf{f})$ Volcano plots of differentially expressed miRNAs in DME vs. CTR (d), DME vs. D (e), and D vs. CTR patient groups (f) computed using Global Mean normalization strategy. Differentially expressed miRNAs found for both normalizations are labeled and indicated as green dots. Fold change cutoff (red lines) was set at 2.5-fold while $p$-values cutoff (blue line) was set at 0.05 based on Student's $t$ test on normally distributed $\triangle \mathrm{CT}$. 
Table 2. List of differentially expressed miRNAs following profiling analyses in AH of DME vs CTR group ( $n=11 ; n=8$ downregulated miRNAs and $n=3$ upregulated miRNAs) and in AH of DME vs. D group ( $n=15$ miRNAs; $n=11$ downregulated miRNAs and $n=4$ upregulated miRNAs).

\begin{tabular}{ccc}
\hline Expressed miRNAs & DME Vs. CTR & DME Vs. D \\
\hline & hsa-let-7c-5p & hsa-let-7c-5p \\
\cline { 2 - 3 } & hsa-miR-193a-5p & hsa-miR-193a-5p \\
\cline { 2 - 3 } Downregulated miRNAs & hsa-miR-19b-3p & hsa-miR-19b-3p \\
\cline { 2 - 3 } & hsa-miR-200b-3p & hsa-miR-200b-3p \\
\cline { 2 - 3 } & hsa-miR-204-5p & hsa-miR-204-5p \\
\cline { 2 - 3 } & hsa-miR-365-3p & hsa-miR-365-3p \\
\cline { 2 - 3 } & hsa-miR-100-5p & hsa-miR-199a-3p \\
\cline { 2 - 3 } & hsa-miR-429 & hsa-miR-20b-5p \\
\cline { 2 - 3 } & & hsa-miR-211-5p \\
\hline \multirow{2}{*}{ Upregulated miRNAs } & hsa-miR-34a-5p & hsa-miR-532-5p \\
\cline { 2 - 3 } & hsa-miR-374-5p & hsa-miR-374-5p \\
\hline
\end{tabular}

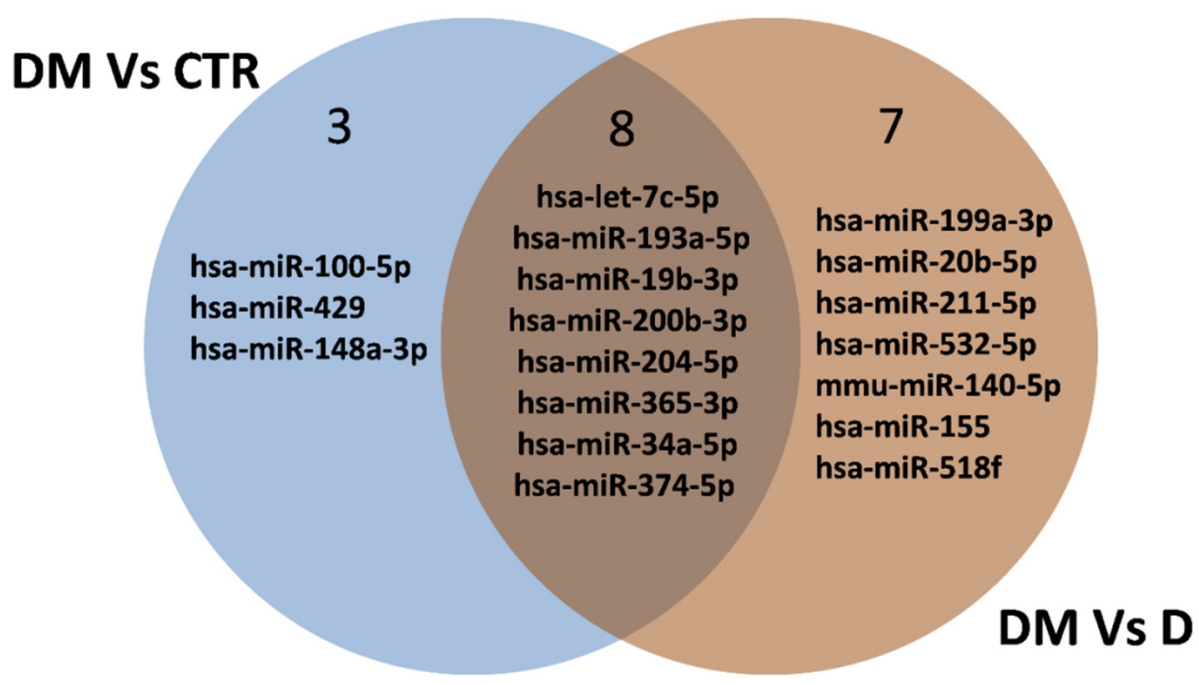

Figure 3. Venn diagram showing the differentially expressed miRNAs among the comparisons of DME vs CTR and of DME vs. D. Eight miRNAs resulted commonly differentially expressed among the comparisons of DME vs. CTR and of DME vs. D $(n=6$ downregulated miRNAs and $n=2$ upregulated miRNAs).

\section{1. miRNAs Downregulation in AH of DME Patients}

Among the differentially expressed miRNAs in DME vs. CTR and in DME vs. D, eight were common to both comparisons, while two were specific to the comparison DME vs. D, thus rendering them specifically altered in the AH of DME patients. Therefore, we focused on single assay validation on this set of 10 miRNAs in the same subjects per group as analyzed in the profiling stage (Figure 4). Four miRNAs out of ten were not validated in single assay since they did not confirm the trend observed in the profiling analysis (miR-204-5p, miR-211-5p, miR-193a-5p and miR-19b-3p) (Figure 4), while six out of ten miRNAs were validated in single assay in the five subjects per group (let-7c-5p, miR-200b-3p, miR-199a-3p, miR-365-3p, miR-34a-5p and miRNA-374-5p) and were then tested in all 
the subjects included in the study ( $n=10 \mathrm{CTR}, n=8 \mathrm{D}, n=12 \mathrm{DME}$ ). miR-34a-5p and miR-374-5p did not pass the validation in a single assay in all subjects (Supplementary Figure S2), while 4 miRNAs out of 10 were confirmed to be differentially expressed in all subjects and resulted all downregulated in DME group vs CTR or vs. D (let-7c-5p, miR-200b-3p, miR-199a-3p and miR-365-3p) (Figure 5).
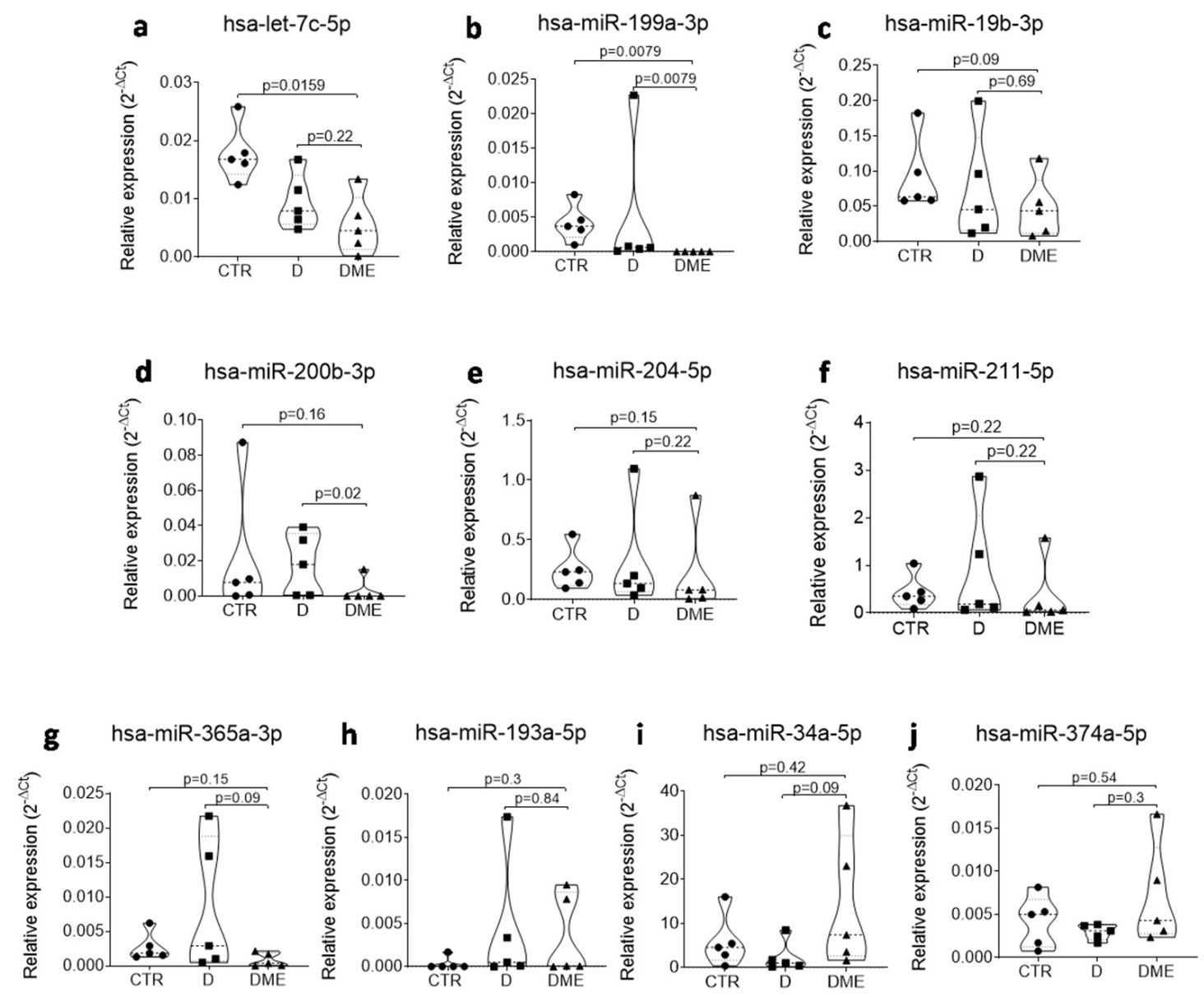

Figure 4. Single assay validation of $n=10$ microRNAs that resulted deregulated in DME vs CTR and/or vs D in AH of the profiling cohort (5 patients per group) of DME vs CTR and vs D patients. Violin plots show the expression and the relative $p$ values of hsa-let-7c-5p (a), hsa-miR-199a-3p (b), hsa-miR-19b-3p (c), hsa-miR-200b-3p (d), hsa-miR-204-5p (e), hsa-miR-211-5p (f), hsa-miR-365-3p (g), hsa-miR-193a-5p (h), hsa-miR-34a-5p (i) and hsa-miR-374a-5p (j). miRNA expression values are reported as $2^{-\Delta C T}$ normalized for the expression of hsa-miR-150-5p. Median values for each group are shown as dotted line alongside with quartiles (smaller dotted lines). $p$-values were considered significant with $p<0.05$ using Mann-Whitney U Test. 

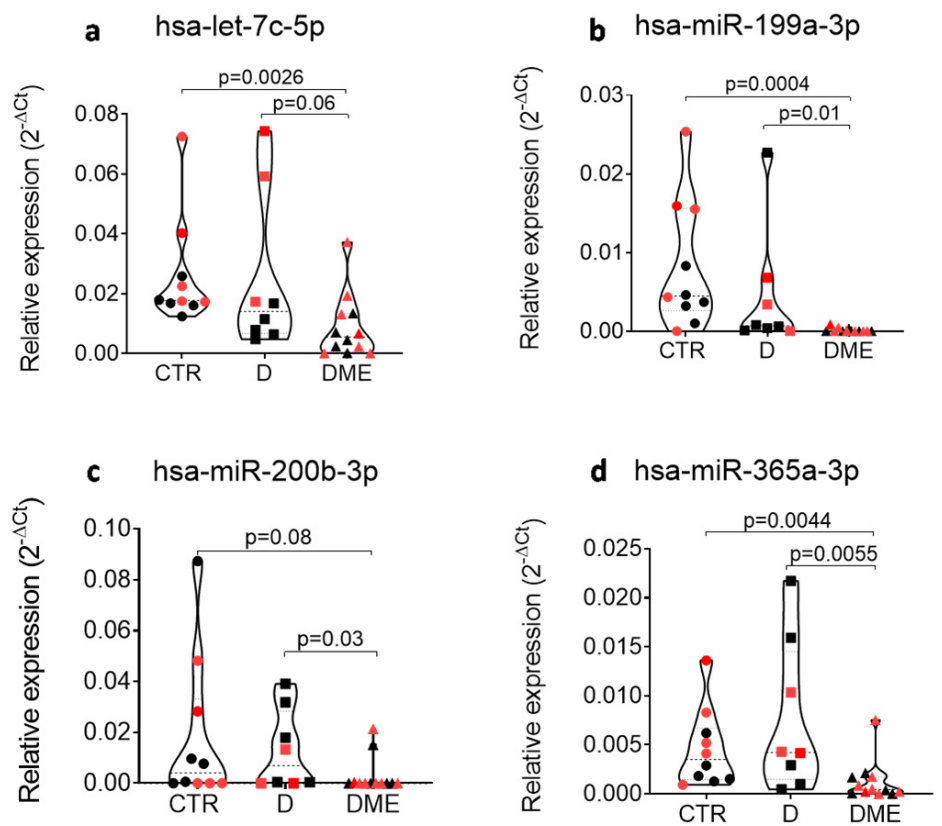

Figure 5. The expression of hsa-let-7c-5p (a), hsa-miR-199a-3p (b), hsa-miR-200b-3p (c) and hsa-miR-365-3p (d) is reduced in AH of DME vs CTR and/or vs D patients after testing for all subjects included in the study $(n=10 \mathrm{CTR}, n=8 \mathrm{D}, n=12 \mathrm{DME})$. Profiling cohort $(n=5 \mathrm{CTR}$, $n=5 \mathrm{D}, n=5 \mathrm{DME})$ is indicated with black dots, while the additional validation cohort $(n=5 \mathrm{CTR}$, $n=3 \mathrm{D}, n=7 \mathrm{DME}$ ) is shown as red dots. microRNA expression values are reported as $2^{-\Delta \mathrm{CT}}$ normalized for the expression of hsa-miR-150-5p. Median values for each group are shown as dotted line alongside with quartiles (smaller dotted lines). $p$-values were considered significant with $p<0.05$ using Mann-Whitney U Test.

\section{2. miR-199a-3p and miR-365-3p Differential Expression in AH is Mirrored in Plasma Samples}

Since we confirmed the differential expression of these four miRNAs in AH samples of DME patients, we hypothesized a systemic alteration of these miRNAs, thus rendering them easily accessible biomarkers. To investigate this hypothesis, we tested them in plasma samples of the same subjects using single assay RT-qPCR. No differential expression for let-7c-5p and miR-200b-3p was observed (Supplementary Figure S3). However, downregulation of miR-199a-3p and miR-365-3p in the plasma of the DME patients was observed (Figure 6), in line with the same reduction observed in the AH samples. However, no significant correlation resulted between the $\mathrm{AH}$ and plasma samples of the same subjects (Supplemental Figure S4).
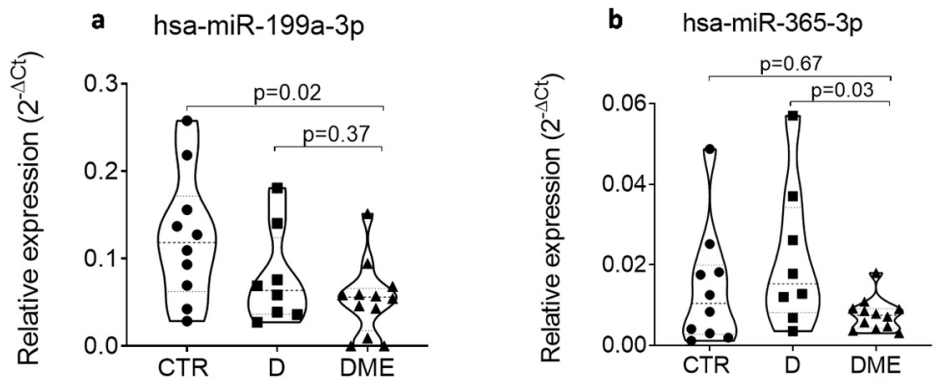

Figure 6. The expression of hsa-miR-199a-3p (a) is reduced in plasma of DME $(n=12)$ vs. CTR $(n=10)$ patients; the expression of hsa-miR-365-3p (b) is reduced in plasma of DME $(n=12)$ vs. D $(n=8)$ patients. miRNA expression is indicated as $2^{-\Delta C T}$ normalized for the expression of hsa-miR-191-5p and hsa-miR-320a-3p. Median values for each group are shown as dotted line alongside with quartiles (smaller dotted lines). $p$-values were considered significant with $p<0.05$ using Mann-Whitney U Test. 


\section{3. miR-199a-3p and miR-200b-3p Are Involved in Retina-Vascular and Epithelial Cells Homeostasis}

In order to functionally correlate AH miRNAs dysregulation to DME pathological alterations, we performed a Gene Ontology (GO) analysis using DAVID 6.8 algorithm on validated target genes of the 4 downregulated miRNAs in the AH of DME patients identified through miRPath 3.0 algorithm. No validated target gene was identified for let-7c-5p, while $n=1614$ validated target genes were identified for overall miR-199a-3p, miR-200b-3p and miR-365-3p (Supplementary File S2). GO bioinformatic analysis results on 1613 genes recognized by DAVID 6.8 algorithm (Supplementary File S2) highlighted that 54 validated target genes of miR-199a-3p, miR-200b-3p and miR-365-3p are significantly involved in 8 pathways that could be potentially implied in the pathogenesis of diabetic macular edema (Table 3), including VEGFA signaling pathway $(n=19$ genes/1613, Fold enrichment $=2.4$, Fisher's exact test corrected $p$ value $=0.00021)$. Interestingly, we also observed that validated target genes of miR-199a-3p, miR-200b-3p and miR-365-3p are involved in establishment of endothelial barrier signaling pathway $(n=9$ genes $/ 1613,0.6 \%$, Fold enrichment $=3.0$, Fisher's exact test corrected $p$ value $=0.0024)$, which includesF11 receptor among the validated target genes of miR-199a-3p.

Accordingly, the correlation between VEGFA expression and both miR-199a-3p and miR-200b-3p was checked measuring VEGFA levels in the AH. Due to lack of residual AH, VEGFA concentrations were measured only in $n=9$ CTRs, $n=6$ Ds and $n=11$ DMEs. However, no correlation between VEGF and either miR-199a-3por miR-200b-3p was found (data not shown).

Since F11 receptor seems to be implicated in the maintenance of retinal pigment epithelium barrier, we then checked whether DME patients showing serous retinal detachment (SRD) at OCT examination showed also miR-199a-3p differential expression levels in AH respect to those not showing any SRD. Five out of 12 DME patients presented DME associated with SRD (42\%). However, miR-199a-3p expression levels were not different between DME patients showing SRD vs. those without SRD (data not shown). 
Table 3. List and details of most important signaling pathways in which validated target genes of miR-200b-3p, miR-365-3p and miR-199a-3p are involved. The first column indicates the biological process in which a specific number (second column) of validated target genes of miR-200b-3p, miR-365-3p and miR-199a-3p are involved. The following columns list the respective $p$ values (third column), fold enrichment (fourth column) and Fisher's exact test corrected $p$ values (fifth column). DLL1: delta like canonical Notch ligand 1; NOTCH1: Notch 1; RBPJ: recombination signal binding protein for immunoglobulin kappa J region; MET: proto-oncogene, receptor tyrosine kinase; FGFR1: fibroblast growth factor receptor 1; KDR: kinase insert domain receptor; THBS1: thrombospondin 1; VEGFA: vascular endothelial growth factor A; AKT1: AKT serine/threonine kinase 1; AMOTL1: angiomotin like 1; NFE2L2: nuclear factor, erythroid 2 like 2; PLCG1: phospholipase C gamma 1; PTGS2: prostaglandin-endoperoxide synthase 2; TGFB1: transforming growth factor beta 1; EPHA2: EPH receptor A2; KLF4: Kruppel like factor 4; EFNA1: ephrin A1; PDCD10: programmed cell death 10; RHOA: ras homolog family member A; F11R: F11 receptor; RAP1B: RAP1B, member of RAS oncogene family; RAP2B: RAP2B, member of RAS oncogene family; RAP2C: RAP2C, member of RAS oncogene family; RAPGEF2: Rap guanine nucleotide exchange factor 2; RAPGEF6: Rap guanine nucleotide exchange factor 6; AFDN: afadin, adherens junction formation factor; MSN: moesin; RDX: radixin; AXL receptor tyrosine kinase(AXL); CRK:CRK proto-oncogene, adaptor protein; NCKAP1: NCK associated protein 1; ACTG1: actin gamma 1; CDC42: cell division cycle 42; ELMO1: engulfment and cell motility 1; ELMO2: engulfment and cell motility 2; FLT1: fms related tyrosine kinase 1; ITGAV: integrin subunit alpha V; NEDD4 neural precursor cell expressed, developmentally down-regulated 4, E3 ubiquitin protein ligase; PAK 2: p21 (RAC1) activated kinase 2; PIK3CB: phosphatidylinositol-4,5-bisphosphate 3-kinase catalytic subunit beta; PIK3R1: phosphoinositide-3-kinase regulatory subunit 1; PRKCB: protein kinase C beta: PTK2: protein tyrosine kinase 2; RAC1: ras-related C3 botulinum toxin substrate 1 (rho family, small GTP binding protein Rac1); AKT serine/threonine kinase 1(AKT1); GREM1: gremlin 1, DAN family BMP antagonist(GREM1); ADAM9: ADAM metallopeptidase domain 9; ETS1: ETS proto-oncogene 1, transcription factor; JUN: Jun proto-oncogene, AP-1 transcription factor subunit; SOX9: SRY-box 9; WNT5A: Wnt family member 5; CAPN7: calpain 7: CLASP1: cytoplasmic linker associated protein 1(CLASP1; DOCK5: dedicator of cytokinesis 5; ZNF580: zinc finger protein 580.

\begin{tabular}{|c|c|c|c|c|c|c|}
\hline Signaling Pathway & Genes Count & Genes \% & Genes ID & $p$-Value & Fold Enrichment & Fisher Exact Test \\
\hline arterial endothelial cell differentiation & 3 & 0.2 & DDL1, NOTCH1, RBPJ & $8.90 \times 10^{-2}$ & 5.8 & $1.10 \times 10^{-2}$ \\
\hline regulation of endothelial cell chemotaxis & 6 & 0.4 & MET, FGFR1, KDR, NOTCH1, THBS1, VEGFA & $1.20 \times 10^{-2}$ & 4.1 & $2.20 \times 10^{-3}$ \\
\hline $\begin{array}{l}\text { positive regulation of blood } \\
\text { vessel endothelial cell migration }\end{array}$ & 8 & 0.5 & AKT1, AMOTL1, NFE2L2, PLCG1, PTGS2,THBS1, TGFB1, VEGFA & $4.30 \times 10^{-3}$ & 3.7 & $8.80 \times 10^{-4}$ \\
\hline $\begin{array}{l}\text { regulation of blood vessels } \\
\text { endothelial cell migration }\end{array}$ & 14 & 0.9 & $\begin{array}{l}\text { AKT1, EPHA2, KLF4, AMOTL1, EFNA1, NOTCH1, NFE2L2, PLCG1, } \\
\text { PDCD10, PTGS2, RHOA, THBS1, TGFB1, VEGFA }\end{array}$ & $3.70 \times 10^{-4}$ & 3.1 & $9.50 \times 10^{-5}$ \\
\hline establishment of endothelial barrier & 9 & 0.6 & $\begin{array}{c}\text { F11R, RAP1B, } \\
\text { RAP2B, RAP2C, } \\
\text { RAPGEF2, RAPGEF6, AFDN, MSN, RDX }\end{array}$ & $8.70 \times 10^{-3}$ & 3 & $2.40 \times 10^{-3}$ \\
\hline $\begin{array}{l}\text { vascular endothelial growth factor } \\
\text { receptor signaling pathway }\end{array}$ & 19 & 1.2 & $\begin{array}{c}\text { AXL, CRK, NCKAP1, } \\
\text { ACTG1, CDC42, ELMO1, ELMO2, FLT1, ITGAV, KDR, NEDD4, PAK2, } \\
\text { PIK3CB, PIK3R1, PRKCB, PTK2, RHOA, RAC1, VEGFA }\end{array}$ & $6.10 \times 10^{-4}$ & 2.4 & $2.10 \times 10^{-4}$ \\
\hline blood vessel endothelial cell migration & 14 & 0.9 & $\begin{array}{l}\text { AKT1, KLF4, AMOTL1, GREM1, KDR, NOTCH1, NFE2L2, PLCG1, } \\
\text { PDCD10, PTGS2, RHOA, THBS1, TGFB1, VEGFA }\end{array}$ & $5.70 \times 10^{-3}$ & 2.3 & $2.10 \times 10^{-3}$ \\
\hline positive regulation of epithelial cell migration & 20 & 1.2 & $\begin{array}{l}\text { ADAM9, AKT1, ETS1, JUN, MET, SOX9, WNT5A, AMOTL1, CAPN7 } \\
\text { CLASP1, , DOCK, FGFR1, KDR, NFE2L2, PLCG1 } \\
\text { PTGS2, THBS1, TGFB1, VEGFA, ZNF580 }\end{array}$ & $1.60 \times 10^{-3}$ & 2.2 & $6.10 \times 10^{-4}$ \\
\hline
\end{tabular}




\section{Discussion}

The peculiarity of the present study starts from the population examined: as a matter of fact, AH miRNAs were evaluated in T2D patients affected by DME. Previous studies evaluating miRNA expression through intraocular sampling are few in number and all but one were conducted on patients affected by PDR, in the context of either type 1 or type 2 diabetes [16-20]. We enrolled only patients showing DME with mild or moderate NPDR in an attempt to minimize the ischemic component and consequently the molecular pathways driven by ischemia. DME associated with PDR would probably have provided new information in any way, but interpretation of the results would have been more difficult due to the ischemic component that characterizes PDR. In fact, based on results in human serum [11,23] and in animal models of diabetic retinopathy [2-7], different stages of DR seem to be associated with different expression of both miRNAs and cytokines. Moreover, we enrolled only T2D patients on the basis of the different etiopathological mechanisms that distinguish T2D from T1D [24]. Cho and colleagues [22] were the only ones to have previously studied miRNA expression in DME in the context of NPDR without specifying if patients were affected by T1D or T2D. Additionally, we compared miRNA expression in DME patients not only with that in patients without diabetes but also with that in patients with diabetes but without DR, in order to verify whether or not diabetes per se could be associated with a particular pattern of intraocular miRNA expression and to study its impact on the DME miRNA profile.

In the present series both $\mathrm{AH}$ and plasma samples were collected at entry from all the patients enrolled. However, since local biomarkers seem to reflect the pathogenic events that are taking place in the retina more directly compared to circulating biomarkers [21,25], we decided to first profile and validate the differential expression of miRNAs in the $\mathrm{AH}$ and then to verify their concomitant differential expression in the plasma.

AH samples have been previously studied to verify different miRNA expression in open-angle glaucoma as compared to the expression in cataract patients [26-29]. Cataract patients also constituted the control group in the present analysis. We detected 107 mature miRNAs in the present CTR population, 97 of which (91.5\%) were shared with the CTR group of Jayaram et al. [26], who also performed their evaluation through microarray analysis.

In patients affected by PDR different miRNA disregulation has been found. Gomaa et al. [16] found miR-200b to be upregulated in both T1D and T2D diabetic patients [16]. Usui-Ouchi et al. [17] identified miR-21 (upregulated in the vitreous of diabetic patients) as potential disease-modifying agent for the development of proliferative disease. Hirota et al. [18] investigated the expression of 168 miRNAs in the vitreous humor of patients with PDR and found that six miRNAs (miR-15a, miR-320a, miR-320b, miR-93, miR-29a and miR-423-5p), related to angiogenesis and fibrosis, are significantly overexpressed in PDR. Mammadzada et al. [19] showed a significant increase in the miRNA-19a, miR-27a, miR-20a and miR-93 expression in PDR patients undergoing PPV as compared to the controls. Recently by next generation sequencing, Chen et al. [20] found seven miRNAs upregulated (miR-150-5p, miR-30c-5p, miR16-2-3p, miR-1827, miR-140-3p and miR-93-5p) and one downregulated (miR-99-5p) in AH of T2D patients with PDR as compared to controls.

In the only study in DME patients, through PCR array of 84 miRNAs, Cho et al. [22] found a significant downregulation of 59 miRNAs in patients as compared to controls. Among the significantly dysregulated miRNAs, five (miR185-5p, hsa-miR-17-5p, hsa-miR-20a-5p, hsa-miR-15b-5p and hsa-miR-15a-5p) were further tested using real-time PCR and all were found to be downregulated.

The four previous studies evaluating miRNA expression in the vitreous of PDR patients relied on patients affected by macular hole as the control group [16-19]. Chen et al. [20], in the evaluation of miRNAs expression of PDR patients, and Cho et al. [22], in the evaluation of miRNA expression in the AH of DME patients, used cataract patients as the control group. Therefore, this is the first study to evaluate intraocular miRNA expression in diabetic patients without DR and/or DME. From our results, diabetes per se does not seem to be characterized by a different AH miRNA profile compared to that of the cataract-only group, since the mature miRNAs detected after profiling was highly similar between 
the two groups. On the contrary, the DME group showed miRNA under-expression compared to both the CTR and D groups (86 miRNA were detected in the DME group vs. 113 in the D group and 107 in the CTR group), which was confirmed in a single assay since miR-200b-3p, let-7c-5p, miR-365-3p and miR-199a-3p were found to be significantly downregulated. As such, different intraocular miRNA expression seems to occur in the diabetic patient only after the process of diabetic retinopathy has begun.

In the present study we show that miR-200b, let-7c, miR-365 and miR-199a-3p were all downregulated in DME patients. If we compare our results with those of Cho et al. [22], in both studies a general downregulation of miRNAs in DME patients as compared to controls is found; however, the single miRNAs identified as downregulated are different. The discrepancies in the differentially expressed miRNAs identified between the two studies might be explained by the different methods of analysis used by Cho et al. Indeed, the authors analyzed the expression of 84 miRNAs among those most studied and already reported to be involved in angiogenesis and inflammation. Moreover, the method of normalization for the evaluation if miRNAs expression is not completely appropriate given the use of the small nucleolar RNU6-6p (which is not a miRNA).

miR-200b, let-7c, miR-365 and miR-199a-3p have already been found to be implicated in DR - miR-200b being the only of the four to have been analyzed on human intraocular samples. However, their protective or aggravating role reported can hardly be compared with their downregulation shown in the present series, since the animal models, being devoid of the macula region, cannot fully mimic the course of DR and the eye's molecular behavior in humans, and since discrepancies in their expression have been reported even when different animal models were used [7,30-32]. The prototype of these previous conflicting results is miR-200b, which has been found to be downregulated in endothelial cells under hyperglycemia in the retinas of STZ-induced diabetic rats and in the sera of DR patients, whereas it is upregulated in the retinas of a genetic model of type 1 diabetes (Akita mice) and the vitreous of PDR patients [7]. In the present seriesmiR-200b-3p is downregulated and not upregulated, as shown by Gomaa et al. [16] in their PDR patients; however, again, we included only patients affected by NPDR. In accordance with our results, both let-7c and mir-365 were found to be implicated in the early stages of the DR animal model but, in contrast to our results, rather than a protective role, they were shown to have an aggravating role: increased vessel tortuosity and decreased pericyte coverage for let-7c and increased oxidative stress for miR-365 [30-32]. In relation to the previously reported expression of miR-365, the discrepancy with the present results is even greater, since the downregulation found in the AH of our patients was also confirmed in their plasma. The only miRNA whose previously reported role is in line with that reported herein is miR-199a-3p. Although implicated in a variety of carcinomas as either a repressor or promoter, miR-199a-3p has been found to be downregulated in the animal model of DR, thus confirming the protective role in both the $\mathrm{AH}$ and plasma reported here [7,33].

GO bioinformatic analysis highlighted that 54 validated target genes of miR-199a-3p, miR-200b-3p and miR-365-3p are significantly involved in eight pathways that could be potentially implied in the pathogenesis of diabetic macular edema. The only soluble mediators associated with these 8 pathways (and therefore measurable in AH) are VEGFA, TGF $\beta 1$ and Gremlin-1, an inhibitor of TGF $\beta$ pathway. Since we recently showed that TGF $\beta 1$ is poorly expressed in the AH $[34,35]$, we decided to measure VEGFA concentration. Additionally, GO analysis identified a direct validated regulation of miR-199a-3p on F11 receptor, which has been shown to be involved in the maintenance of the retinal pigment epithelium barrier [36]. However, no correlation between VEGFA and either miR-199a-3p or miR-200b-3pAH levels was found. This is not surprising, since anti-VEGFA agents show inefficacy in around half of DME patients notwithstanding intensive injection regimens and, as reported by Aiello et al. [37], 36\% of diabetic patients with PDR had undetectable levels of VEGFA in the vitreous fluid $[3,4,21]$. F11R/JAM-A is a tight junction protein that is important for platelet and leukocyte interactions with the epithelium and endothelium and has been found to be expressed by the retinal pigment epithelium [36]. Accordingly, it might be implicated in the regulation of this important physiological barrier and in the occurrence of macular edema, and in particular with the DME subtype 
associated with SRD. Although the pathogenetic theories at the basis of SRD occurrence are different, the role of RPE in retinal fluid homeostasis seems to be clinically confirmed by the results of the present series, since the DME patients showed a SRD incidence of $42 \%$, which is in the high range compared to the $15 \%-45 \%$ reported in the literature [38-40]. However, we failed to demonstrate a significant differential expression of each of the downregulated miRNAs, including miR-199a-3p, between patients with and without SRD.

The site of intraocular sampling may represent a potential limitation of the present study. Sampling of vitreous humor could be more informative for patients affected by DR and DME; however, due to ethical reasons, $\mathrm{AH}$ is nowadays considered the most appropriate biological sample for the investigation of intraocular biomarkers in DME. The formation of cataract might have had an effect on our results in CTR and D patients; however, no difference in age was present between the three groups.

In conclusion, different intraocular miRNA expression seems to occur in diabetic patients only after the process of diabetic retinopathy has begun. In patients affected by NPDR complicated by DME, miR-200b-3p, let-7c-5p, miR-365-3p and miR-199a-3p were downregulated in the AH, and miR-365-3p and miR-199a-3p were also downregulated in the plasma. Additional studies are needed to understand the complicated molecular interplay occurring in DME and to verify the role of miR-199a-3p in relation to the retinal pigment epithelial barrier.

\section{Materials and Methods}

\subsection{Subjects}

The present study was performed on three groups of patients: $n=12$ T2D patients with DR complicated by DME (DME group), $n=8$ T2D patients without any sign of DR (D group), and $n=10$ non-diabetic patients (CTR group). All eyes were examined and treated between September 2016 and March 2018 at the Ophthalmology Unit of the Department of Medicine, Surgery and Neuroscience, Siena University Hospital, Siena, Italy, following approval by the Institutional review board C.E.A.V.S.E. (Ethical Committee for clinical spermientation, South-Eastern Tuscany) (identification code MIRNADR16, approval date 16 May 2016). The study complied with the Declaration of Helsinki and patients were treated after being informed of the nature, purpose, implications and risks of the treatment and after having signed a consent form.

Patient demographics were recorded, including length of diabetic history, glycosylated hemoglobin $(\mathrm{HbA1c})$, body mass index (BMI) and systemic medications. Ophthalmological examination included best-corrected visual acuity (BCVA) by Snellen chart recording (and conversion to logMAR for statistical analysis) and intraocular pressure measurement, anterior segment slit lamp examination, dilated fundus examination with slit-lamp biomicroscopy and indirect ophthalmoscopy, as well as spectral-domain OCT central subfield thickness (CST) (Cirrus HD-OCT; Carl Zeiss Meditec AG, Jena, Germany). The baseline severity of DR and the diagnosis of center-involving macular edema among the eyes enrolled were evaluated following clinical and OCT examinations according to the International Council of Ophthalmology recommendations [1].

The inclusion criteria were: (i) for the DME group—being affected by T2D complicated by mild to moderate NPDR associated with treatment-naive center-involving DME with a CST of $>310 \mu \mathrm{m}$; (ii) for the D group—being affected by T2D without signs of DR and/or DME; (iii) for the CTR group —not being affected by T2D. All patients included were phakic with a mild to moderate cataract. For the DME group whenever DME was bilaterally present, the eye with a higher CST determined by SD-OCT was selected as the study eye.

The exclusion criteria were: race other than Caucasian; types of diabetes other than T2D and presence or history of underlying connective tissue, inflammatory or malignant disease; presence of hepatic insufficiency, renal impairment and infectious diseases; ischemic heart attack in the previous 12 months; diabetic neuropathy; being affected by severe non-proliferative or proliferative DR or by any other ocular disease except mild to moderate cataract for the DME group; being affected by any other 
ocular disease except mild to moderate cataract for the D group and CTR groups; prior intraocular surgery of any kind, including cataract surgery; and history of any intravitreal injection, ocular trauma or laser treatment.

\subsection{AH and Plasma Sample Collection and Processing}

Approximately 50 to $100 \mu \mathrm{L}$ of $\mathrm{AH}$ were collected from each patient through a clear corneal paracentesis using a 30-gauge needle just before the intravitreal injection (DME group) or prior to placement of the initial cataract incision (D and CTR group). AH samples were collected in $0.5 \mathrm{~mL}$ nuclease-free microtubes, stored on-ice and further processed within $2 \mathrm{~h}$ from collection. Sample collection was atraumatic in all cases, thereby eliminating the risk of contamination with blood or cellular debris. Eventual cells/cellular debris contamination was prevented by centrifuging AH samples at $1200 \times \mathrm{g}$ for $20 \mathrm{~min}$ at $10{ }^{\circ} \mathrm{C}$. Supernatant was then transferred to another tube and stored at $-80^{\circ} \mathrm{C}$ until ready for RNA extraction.

A SOP was followed to collect plasma samples as previously described [41]. Blood was collected in BD Vacutainer $\mathrm{K}_{2}$-EDTA tubes (BD Biosciences, San Jose, CA, USA), inverted 5 times and stored upright at room temperature until ready for processing. Blood samples were processed within $2 \mathrm{~h}$ from blood draw by centrifugation at $1800 \times g$ for $10 \mathrm{~min}$ at room temperature; collected plasma was further centrifuged at $1200 \times g$ for $20 \mathrm{~min}$ at $10^{\circ} \mathrm{C}$ in order to remove contaminant cells and cellular debris. Finally, plasma samples were aliquoted and subsequently stored at $-80^{\circ} \mathrm{C}$. Hemolyzed plasma samples were excluded from the study.

\subsection{RNA Extraction}

Total RNA, including small RNAs $<200 \mathrm{nt}$, was extracted from $50 \mu \mathrm{L}$ of $\mathrm{AH}$ or $200 \mu \mathrm{L}$ of plasma samples using MicroRNA miRNeasy Mini extraction kit (Qiagen, Hilden, Germany) with some modifications. AH and plasma samples were thawed on ice and centrifuged at $3000 \times g$ for 5 min at $4{ }^{\circ} \mathrm{C}$ to completely remove contaminant cellular debris. A total of $50 \mu \mathrm{L}$ of $\mathrm{AH}$ and $200 \mu \mathrm{L}$ of plasma from each patient were diluted in up to $400 \mu \mathrm{L}$ in nuclease-free water to avoid protein aggregates. Samples were then lysed by adding 3 volumes of Trizol LS (Life Technologies, Carlsbad, CA, USA) and finally eluted in $30 \mu \mathrm{L}$ of nuclease-free water.

\subsection{TaqMan Array miRNA Expression Profiling}

TaqMan MicroRNA Human Array Panel A platform (Life Technologies, Carlsbad, CA, USA) was adopted to profile the expression of 378 miRNAs in total RNA extracted from AH samples. RNA was reverse-transcribed using Megaplex RT primers Pool-A (Life Technologies, Carlsbad, CA, USA). Briefly, $3 \mu \mathrm{L}$ of extracted RNA from $50 \mu \mathrm{L}$ of each AH sample were added to $0.8 \mu \mathrm{L}$ of $10 \times$ Megaplex RT Primers, $0.2 \mu \mathrm{L}$ of $100 \mathrm{mMdNTPs}, 1.5 \mu \mathrm{L}$ of $50 \mathrm{U} / \mu \mathrm{L}$ Multiscribe RT, $0.8 \mu \mathrm{L}$ of $10 \times$ RT Buffer, $0.9 \mu \mathrm{L}$ of $25 \mathrm{mM} \mathrm{MgCl} 2,0.1 \mu \mathrm{L}$ of $20 \mathrm{U} / \mu \mathrm{L}$ RNAse inhibitor and $0.2 \mu \mathrm{L}$ of nuclease-free $\mathrm{H}_{2} \mathrm{O}$. The product of this reaction was incubated for 40 cycles at $16{ }^{\circ} \mathrm{C}$ for $2 \mathrm{~min}, 42^{\circ} \mathrm{C}$ for $1 \mathrm{~min}$ and $50{ }^{\circ} \mathrm{C}$ for $1 \mathrm{~s}$, and then at $85{ }^{\circ} \mathrm{C}$ for $5 \mathrm{~min}$. Afterwards, the synthesized cDNA was pre-amplified using Megaplex Preamp primers Pool-A. $2.5 \mu \mathrm{L}$ of cDNA from each sample were added to $12.5 \mu \mathrm{L}$ of $2 \times$ TaqMan Preamp Master Mix, 2.5 $\mu \mathrm{L}$ of $10 \times$ Preamp Primers A V.2.1 and 7.5 $\mu \mathrm{L}$ of $\mathrm{H}_{2} \mathrm{O}$. The product of this reaction was incubated at $95^{\circ} \mathrm{C}$ for $10 \mathrm{~min}$, at $55^{\circ} \mathrm{C}$ for $2 \mathrm{~min}$ and at $72{ }^{\circ} \mathrm{C}$ for $2 \mathrm{~min}$, then for 12 cycles at $95^{\circ} \mathrm{C}$ for $15 \mathrm{~s}$ and $60^{\circ} \mathrm{C}$ for $4 \mathrm{~min}$ and, finally, at $99^{\circ} \mathrm{C}$ for $10 \mathrm{~min}$. Finally, the preamplified cDNA was diluted $1: 4$ in $0.1 \times$ Tris-EDTA pH8.0 to obtain a final volume of $100 \mu \mathrm{L}$.

The reaction mix for each microfluidic card was prepared by adding $360 \mu \mathrm{L}$ of $\mathrm{H}_{2} \mathrm{O}$ and $450 \mu \mathrm{L}$ of TaqMan Universal PCR Master Mix $2 \times$ to $90 \mu \mathrm{L}$ of diluted and pre-amplified cDNA. The product of this reaction was incubated at $95^{\circ} \mathrm{C}$ for $10 \mathrm{~min}$, followed by 40 cycles of $95^{\circ} \mathrm{C}$ for $15 \mathrm{~s}$ and $60^{\circ} \mathrm{C}$ for $1 \mathrm{~min}$. The Real-Time PCR instrument ViiA7 (Life Technologies, Carlsbad, CA, USA) was used to perform the reactions. 
Resulting data were analyzed and exported using Expression Suite 1.2.1 software (Life Technologies, Carlsbad, CA, USA). Comparative analyses among DME, D and CTR groups was performed by using $2^{-\Delta \Delta C t}$ method following normalization with both Global Mean Normalization (GMN) method and most stable housekeeping miRNAs in $\mathrm{AH}$.

The expression of 10 differentially expressed miRNAs obtained from the profiling in the comparison between the DME and D groups and the DME and CTR groups was validated on the same 5 subjects per group used for the profiling through a single assay RT-qPCR using TaqMan miRNA chemistry. Among the 10 miRNAs chosen, only the miRNAs which confirmed to be differentially expressed in a single assay were further analyzed in single assays in the AH of the whole population. Only the miRNAs validated in the AH of all subjects were then analyzed in a single assay in plasma from the same subjects. Differential miRNA expression was calculated by relative quantification using the comparative Ct method, with normalization performed using miR-150-5p, miR-373-3p and miR-381-3p. The results were expressed as the fold change in DME AH group compared with AH from CTR and D groups, representing the ratio of the mean normalized expression values of both groups.

\subsection{Selection of Housekeeping miRNAs for AH Samples}

To establish candidate endogenous miRNAs to be exploited as reference normalizers we adopted a data-driven approach based on a combination of three different algorithms as described by Marabita et al. [42]. Each of the three algorithms generated a stability score such that a smaller score corresponds to higher expression stability that we combined into the summarized stability score (SSS) to select top candidate miRNAs in all the methods. We considered only miRNAs with complete observations since we wanted to select miRNAs serving as endogenous controls which were abundantly and stably detected in all samples. In more details, to measure the stability of miRNA expression among DME, D and CTR groups we used geNorm [43] and Normfindern [44] which were implemented into the R/Bioconductor package NormqPCR [45] and a CV-based score. For each miRNA, geNorm calculates the pairwise variation $(V)$ with all other miRNAs across the samples and defines a stability score $(M)$ as the average $\mathrm{V}$ of a particular miRNA with all other control miRNAs. Genes with the lowest $M$ values have the most stable expression. Instead, Normfinder is a model-based approach which estimates the intergroup and intragroup variations of a miRNA, and then combines them into a stability value $(r h o)$. Then, for each miRNA we calculated the coefficient of variation $(\mathrm{CV})$ as its standard deviation across samples divided by the mean and scaled it by the sum of all CVs for each sample ( $C V$ score). Lastly, the SSS was calculated as the three-dimension Euclidean distance from the origin, i.e.,

$$
S S S=\sqrt{\text { mean } M^{2}+r h o^{2}+C V s c o r e^{2}}
$$

In so doing, SSS combined the results from all the methods and allowed to reveal the most stable housekeeping miRNAs (Figure 1c). The stability scores generated by each algorithm for the top 10 candidate miRNAs are shown in Supplementary Figure S1 while the scores full list is reported in Supplementary File S1.

\section{6. miRNAs Single Assay RT-qPCR}

RNA was reverse-transcribed employing Custom RT primers pool and preamplified using Custom Preamp primers pool. Briefly, $5 \mu \mathrm{L}$ each RT or TM primer (Supplementary Table S1) was diluted in a total volume of $500 \mu \mathrm{L}$ of Tris-EDTA $1 \times$ and used for RT or preamplification reaction. Then, $3 \mu \mathrm{L}$ of extracted RNA were added to $6 \mu \mathrm{L}$ of custom primers pool, $0.30 \mu \mathrm{L} 100 \mathrm{mMdNTPs}, 3 \mu \mathrm{L}$ of $50 \mathrm{U} / \mu \mathrm{L}$ Multiscribe RT, $1.50 \mu \mathrm{L} 10 \times$ RT Buffer, $0.19 \mu \mathrm{L} 20 \mathrm{U} / \mu \mathrm{L}$ RNase inhibitor and $1.01 \mu \mathrm{L} \mathrm{H}_{2} \mathrm{O}$. The reaction product was incubated at $16^{\circ} \mathrm{C}$ for $30 \mathrm{~min}, 42^{\circ} \mathrm{C}$ for $30 \mathrm{~min}$ and then at $85^{\circ} \mathrm{C}$ for $5 \mathrm{~min}$. Afterwards, the synthesized cDNA was preamplified using Custom Preamp primer pool: $2.5 \mu \mathrm{L}$ of cDNA from each sample were added to $12.5 \mu \mathrm{L} 2 \times$ TaqMan Preamp Master Mix, $3.75 \mu \mathrm{L} 10 \times$ Custom Preamp primers and $6.75 \mu \mathrm{L}$ nuclease-free $\mathrm{H}_{2} \mathrm{O}$. The reaction was incubated at $95^{\circ} \mathrm{C}$ for $10 \mathrm{~min}$, at $55^{\circ} \mathrm{C}$ for $2 \mathrm{~min}$ and at 
$72{ }^{\circ} \mathrm{C}$ for $2 \mathrm{~min}$, then for 12 cycles at $95^{\circ} \mathrm{C}$ for $15 \mathrm{~s}$ and $60^{\circ} \mathrm{C}$ for $4 \mathrm{~min}$ and, finally, at $99^{\circ} \mathrm{C}$ for $10 \mathrm{~min}$. In each well, $5 \mu \mathrm{L}$ of preamplified cDNA (diluted 1:8 in Tris-EDTA $0.1 \times$ ) were added to $15 \mu \mathrm{L}$ reaction mix composed of $10 \mu \mathrm{L}$ TaqMan Universal Master Mix, $1 \mu \mathrm{L}$ of TaqMan miRNA expression assay and $4 \mu \mathrm{L}$ of nuclease-free $\mathrm{H}_{2} \mathrm{O}$. The reaction was incubated at $95^{\circ} \mathrm{C}$ for $10 \mathrm{~min}$, followed by 40 cycles at $95^{\circ} \mathrm{C}$ for $15 \mathrm{~s}$ and at $60^{\circ} \mathrm{C}$ for 1 minute. ViiA7 Real-Time PCR instrument was used to analyze the reactions while Expression Suite 2.1 software was used to evaluate the amplification plot efficiencies and to analyze expression data. Analysis was performed using $2^{-\Delta \mathrm{Ct}}$ method upon normalization of raw $\mathrm{Ct}$ using expression level of miR-150-5p (for AH samples) which was the most stably expressed among those identified in the profiling stage (Supplementary Figure S1). miRNA miR-320a-3p and miR-191-5p expression levels were adopted to perform normalization in plasma samples as previously shown [46].

\subsection{Bioinformatic Analysis}

miRPath 3.0 algorithm was used to identify validated target genes of the validated differentially expressed miRNAs and Gene Ontology (GO) analysis was performed using DAVID 6.8 algorithm to identify biological processes and specific pathways putatively implicated in the pathogenesis of DME in which the validated target genes of the differentially expressed miRNAs are involved.

\subsection{Proquantum High-Sensitivity Immunoassay}

AH VEGFA concentration was evaluated in $n=9$ CTR subjects, $n=6 \mathrm{D}$ and $n=11$ DME patients through specific Proquantum VEGFA immunoassay following the manufacturer's instructions. Briefly, $5 \mu \mathrm{L}$ of each AH sample diluted 1:3 in assay dilution buffer were added to $5 \mu \mathrm{L}$ of antibody-conjugate mix (calculated for 26 samples: 12 of antibody-conjugate A, $12 \mu \mathrm{L}$ of antibody-conjugate B and $696 \mu \mathrm{L}$ of antibody-conjugate dilution buffer) and incubated $1 \mathrm{~h} \mathrm{RT}$. Then, $40 \mu \mathrm{L}$ of qPCR reaction mix were added to each well and RT-qPCR was performed at the following temperatures: $25^{\circ} \mathrm{C}$ for $20 \mathrm{~min}$, $95^{\circ} \mathrm{C}$ for $2 \mathrm{~min}, 40$ cycles $\left(95^{\circ} \mathrm{C} 1 \mathrm{~s}, 60^{\circ} \mathrm{C} 20 \mathrm{~s}\right)$. The Real-Time PCR instrument ViiA7 was used to perform the reactions. The subsequent analysis was performed through Proquantum online app (apps.thermofisher.com/apps/proquantum).

\subsection{Statistical Analysis}

Data are reported as means or median $\pm \mathrm{SD}$; sample value distribution analysis was performed for all parameters using the D'Agostino-Pearson normality test. The non-parametric Mann-Whitney $\mathrm{U}$ Test or Student's $t$-test was adopted for $p$ value evaluation according to the distribution of values; a $p$ value $\leq 0.05$ was considered as statistically significant. MicroRNA expression levels were reported as normalized $2^{-\Delta \mathrm{Ct}}$ values compared among the selected groups using the Mann-Whitney $\mathrm{U}$ test $(p$ value $\leq 0.05)$.

Supplementary Materials: Supplementary materials can be found at http://www.mdpi.com/1422-0067/21/19/ 7328/s1.

Author Contributions: Conceptualization, F.D. and G.M.T.; Data curation, G.E.G., G.N., L.N., N.B., M.P., T.B. and E.D.B.; Formal analysis, G.S., R.D., M.O. and F.G.; Funding acquisition, F.D. and G.M.T.; Investigation, G.S., M.F. and G.M.T.; Methodology, G.S., M.O., F.G., F.D. and G.M.T.; Project administration, F.D. and G.M.T.; Resources, M.F., F.D. and G.M.T.; Software, G.S. and R.D.; Supervision, F.D. and G.M.T.; Validation, G.S., C.M.E., M.O. and F.G.; Visualization, G.E.G., G.S., C.M.E., G.N., L.N., N.B., R.D., M.P., T.B., E.D.B., M.F., M.O., F.G., F.D. and G.M.T.; Writing—original draft, G.E.G. and G.M.T.; Writing—review and editing, C.M.E., F.D. and G.M.T. All authors have read and agreed to the published version of the manuscript.

Funding: This research was funded by I.Ri.Fo.R (Istituto per la Ricerca, la Formazione e la Riabilitazione Onlus), grant number prot.2747, 08/08/2019.

Conflicts of Interest: The authors declare no conflict of interest. The funders had no role in the design of the study; in the collection, analyses, or interpretation of data; in the writing of the manuscript, or in the decision to publish the results. 


\section{Abbreviations}

$\begin{array}{ll}\text { miRNA } & \text { MicroRNA } \\ \text { AH } & \text { Aqueous humor } \\ \text { T2D } & \text { Type 2 diabetes mellitus } \\ \text { DR } & \text { Diabetic retinopathy } \\ \text { DME } & \text { Diabetic macular edema } \\ \text { CTR } & \text { Control } \\ \text { GO } & \text { Gene ontology } \\ \text { T1D } & \text { Type 1 diabetes mellitus } \\ \text { VEGFA } & \text { Vascular endothelial growth factor A } \\ \text { PDR } & \text { Proliferative diabetic retinopathy } \\ \text { NPDR } & \text { Non-proliferative diabetic retinopathy } \\ \text { HbA1c } & \text { Glycosylated hemoglobin } \\ \text { BMI } & \text { Body mass index } \\ \text { BCVA } & \text { Best-corrected visual acuity } \\ \text { LogMAR } & \text { Logarithm of the minimum angle of resolution } \\ \text { OCT } & \text { Optical coherence tomography } \\ \text { CST } & \text { Central subfield thickness } \\ \text { SOP } & \text { Standard operating procedure } \\ \text { Ct } & \text { Cycle threshold } \\ \text { SRD } & \text { Serous retinal detachment } \\ \text { TGF } \beta 1 & \text { Transforming growth factor } \beta 1 \\ & \end{array}$

\section{References}

1. International Council of Ophthalmology. ICO Guidelines for Diabetic Eye Care. Available online: http: //www.icoph.org/downloads/ICOGuidelinesforDiabeticEyeCare.pdf (accessed on 1 December 2017).

2. Daruich, A.; Matet, A.; Moulin, A.; Kowalczuk, L.; Nicolas, M.; Sellam, A.; Rothschild, P.R.; Omri, S.; Gélizé, E.; Jonet, L.; et al. Mechanisms of macular edema: Beyond the surface. Prog. Retin. Eye Res. 2018, 63, 20-68. [CrossRef] [PubMed]

3. Hillier, R.J.; Ojaimi, E.; Wong, D.T.; Mak, M.Y.K.; Berger, A.R.; Kohly, R.P.; Kertes, P.J.; Forooghian, F.; Boyd, S.R.; Eng, K.; et al. Aqueous Humor Cytokine Levels and Anatomic Response to Intravitreal Ranibizumab in Diabetic Macular Edema. JAMA Ophthalmol. 2018, 136, 382-388. [CrossRef] [PubMed]

4. Figueras-Roca, M.; Sala-Puigdollers, A.; Zarranz-Ventura, J.; Alba-Linero, C.; Alforja, S.; Esquinas, C.; Molins, B.; Adán, A. Anatomic Response to Intravitreal Dexamethasone Implant and Baseline Aqueous Humor Cytokine Levels in Diabetic Macular Edema. Investig. Ophthalmol. Vis. Sci. 2019, 60, 1336-1343. [CrossRef] [PubMed]

5. Felfeli, T.; Juncal, V.R.; Hillier, R.J.; Mak, M.Y.K.; Wong, D.T.; Berger, A.R.; Kohly, R.P.; Kertes, P.J.; Eng, K.T.; Boyd, S.R.; et al. Aqueous Humor Cytokines and Long-Term Response to Anti-Vascular Endothelial Growth Factor Therapy in Diabetic Macular Edema. Am. J. Ophthalmol. 2019, 206, 176-183. [CrossRef] [PubMed]

6. Wu, Y.; Jia, K.; Wu, H.; Sang, A.; Wang, L.; Shi, L.; Jiang, K.; Dong, J. A comprehensive competitive endogenous RNA network pinpoints key molecules in diabetic retinopathy. Mol. Med. Rep. 2019, 19, 851-860. [CrossRef]

7. Gong, Q.; Su, G. Roles of miRNAs and long noncoding RNAs in the progression of diabetic retinopathy. Biosci. Rep. 2017, 37. [CrossRef]

8. Martinez, B.; Peplow, P.V. MicroRNAs as biomarkers of diabetic retinopathy and disease progression. Neural Regen. Res. 2019, 14, 1858-1869. [CrossRef]

9. Mastropasqua, R.; Toto, L.; Cipollone, F.; Santovito, D.; Carpineto, P.; Mastropasqua, L. Role of microRNAs in the modulation of diabetic retinopathy. Prog. Retin. Eye Res. 2014, 43, 92-107. [CrossRef]

10. Zhou, H.; Peng, C.; Huang, D.S.; Liu, L.; Guan, P. microRNA Expression Profiling Based on Microarray Approach in Human Diabetic Retinopathy: A Systematic Review and Meta-Analysis. DNA Cell Biol. 2020, 39, 441-450. [CrossRef] 
11. Mazzeo, A.; Beltramo, E.; Lopatina, T.; Gai, C.; Trento, M.; Porta, M. Molecular and functional characterization of circulating extracellular vesicles from diabetic patients with and without retinopathy and healthy subjects. Exp. Eye Res. 2018, 176, 69-77. [CrossRef]

12. Ma, J.; Wang, J.; Liu, Y.; Wang, C.; Duan, D.; Lu, N.; Wang, K.; Zhang, L.; Gu, K.; Chen, S.; et al. Comparisons of serum miRNA expression profiles in patients with diabetic retinopathy and type 2 diabetes mellitus. Clin. Sao Paulo Braz. 2017, 72, 111-115. [CrossRef]

13. Liu, H.N.; Cao, N.J.; Li, X.; Qian, W.; Chen, X.L. Serum microRNA-211 as a biomarker for diabetic retinopathy via modulating Sirtuin 1. Biochem. Biophys. Res. Commun. 2018, 505, 1236-1243. [CrossRef] [PubMed]

14. Qing, S.; Yuan, S.; Yun, C.; Hui, H.; Mao, P.; Wen, F.; Ding, Y.; Liu, Q. Serum miRNA biomarkers serve as a fingerprint for proliferative diabetic retinopathy. Cell. Physiol. Biochem. Int. J. Exp. Cell. Physiol. Biochem. Pharmacol. 2014, 34, 1733-1740. [CrossRef] [PubMed]

15. Zampetaki, A.; Willeit, P.; Burr, S.; Yin, X.; Langley, S.R.; Kiechl, S.; Klein, R.; Rossing, P.; Chaturvedi, N.; Mayr, M. Angiogenic microRNAs Linked to Incidence and Progression of Diabetic Retinopathy in Type 1 Diabetes. Diabetes 2016, 65, 216-227. [CrossRef] [PubMed]

16. Gomaa, A.R.; Elsayed, E.T.; Moftah, R.F. MicroRNA-200b Expression in the Vitreous Humor of Patients with Proliferative Diabetic Retinopathy. Ophthalmic Res. 2017, 58, 168-175. [CrossRef] [PubMed]

17. Usui-Ouchi, A.; Ouchi, Y.; Kiyokawa, M.; Sakuma, T.; Ito, R.; Ebihara, N. Upregulation of Mir-21 Levels in the Vitreous Humor Is Associated with Development of Proliferative Vitreoretinal Disease. PLoS ONE 2016, 11, e0158043. [CrossRef] [PubMed]

18. Hirota, K.; Keino, H.; Inoue, M.; Ishida, H.; Hirakata, A. Comparisons of microRNA expression profiles in vitreous humor between eyes with macular hole and eyes with proliferative diabetic retinopathy. Graefes Arch. Clin. Exp. Ophthalmol. Albrecht Graefes Arch. Klin. Exp. Ophthalmol. 2015, 253, 335-342. [CrossRef] [PubMed]

19. Mammadzada, P.; Bayle, J.; Gudmundsson, J.; Kvanta, A.; André, H. Identification of Diagnostic and Prognostic microRNAs for Recurrent Vitreous Hemorrhage in Patients with Proliferative Diabetic Retinopathy. J. Clin. Med. 2019, 8, 2217. [CrossRef] [PubMed]

20. Chen, S.; Yuan, M.; Liu, Y.; Zhao, X.; Lian, P.; Chen, Y.; Liu, B.; Lu, L. Landscape of microRNA in the aqueous humour of proliferative diabetic retinopathy as assessed by next-generation sequencing. Clin. Experiment. Ophthalmol. 2019, 47, 925-936. [CrossRef] [PubMed]

21. Vujosevic, S.; Simó, R. Local and Systemic Inflammatory Biomarkers of Diabetic Retinopathy: An Integrative Approach. Investig. Ophthalmol. Vis. Sci. 2017, 58, BIO68-BIO75. [CrossRef]

22. Cho, H.; Hwang, M.; Hong, E.H.; Yu, H.; Park, H.; Koh, S.; Shin, Y.U. Micro- RNAs in the aqueous humour of patients with diabetic macular oedema. Clin. Experiment. Ophthalmol. 2020, 48, 624-635. [CrossRef] [PubMed]

23. Pastukh, N.; Meerson, A.; Kalish, D.; Jabaly, H.; Blum, A. Serum miR-122 levels correlate with diabetic retinopathy. Clin. Exp. Med. 2019, 19, 255-260. [CrossRef] [PubMed]

24. Skyler, J.S.; Bakris, G.L.; Bonifacio, E.; Darsow, T.; Eckel, R.H.; Groop, L.; Groop, P.H.; Handelsman, Y.; Insel, R.A.; Mathieu, C.; et al. Differentiation of Diabetes by Pathophysiology, Natural History, and Prognosis. Diabetes 2017, 66, 241-255. [CrossRef] [PubMed]

25. Ragusa, M.; Barbagallo, C.; Statello, L.; Caltabiano, R.; Russo, A.; Puzzo, L.; Avitabile, T.; Longo, A.; Toro, M.D.; Barbagallo, D.; et al. miRNA profiling in vitreous humor, vitreal exosomes and serum from uveal melanoma patients: Pathological and diagnostic implications. Cancer Biol. Ther. 2015, 16, 1387-1396. [CrossRef] [PubMed]

26. Jayaram, H.; Phillips, J.I.; Lozano, D.C.; Choe, T.E.; Cepurna, W.O.; Johnson, E.C.; Morrison, J.C.; Gattey, D.M.; Saugstad, J.A.; Keller, K.E. Comparison of MicroRNA Expression in Aqueous Humor of Normal and Primary Open-Angle Glaucoma Patients Using PCR Arrays: A Pilot Study. Investig. Ophthalmol. Vis. Sci. 2017, 58, 2884-2890. [CrossRef] [PubMed]

27. Dunmire, J.J.; Lagouros, E.; Bouhenni, R.A.; Jones, M.; Edward, D.P. MicroRNA in aqueous humor from patients with cataract. Exp. Eye Res. 2013, 108, 68-71. [CrossRef] [PubMed]

28. Tanaka, Y.; Tsuda, S.; Kunikata, H.; Sato, J.; Kokubun, T.; Yasuda, M.; Nishiguchi, K.M.; Inada, T.; Nakazawa, T. Profiles of extracellular miRNAs in the aqueous humor of glaucoma patients assessed with a microarray system. Sci. Rep. 2014, 4, 5089. [CrossRef] 
29. Wecker, T.; Hoffmeier, K.; Plötner, A.; Grüning, B.A.; Horres, R.; Backofen, R.; Reinhard, T.; Schlunck, G. MicroRNA Profiling in Aqueous Humor of Individual Human Eyes by Next-Generation Sequencing. Investig. Ophthalmol. Vis. Sci. 2016, 57, 1706-1713. [CrossRef]

30. Zhou, Q.; Frost, R.J.A.; Anderson, C.; Zhao, F.; Ma, J.; Yu, B.; Wang, S. let-7 Contributes to Diabetic Retinopathy but Represses Pathological Ocular Angiogenesis. Mol. Cell. Biol. 2017, 37. [CrossRef]

31. Zheng, K.; Wang, N.; Shen, Y.; Zhang, Z.; Gu, Q.; Xu, X.; Qin, Q.; Liu, Y. Pro-apoptotic effects of micro-ribonucleic acid-365 on retinal neurons by targeting insulin-like growth factor-1 in diabetic rats: An in vivo and in vitro study. J. Diabetes Investig. 2018, 9, 1041-1051. [CrossRef]

32. Wang, J.; Zhang, J.; Chen, X.; Yang, Y.; Wang, F.; Li, W.; Awuti, M.; Sun, Y.; Lian, C.; Li, Z.; et al. miR-365 promotes diabetic retinopathy through inhibiting Timp3 and increasing oxidative stress. Exp. Eye Res. 2018, 168, 89-99. [CrossRef] [PubMed]

33. Wang, H.; Wang, Z.; Tang, Q. Reduced expression of microRNA-199a-3p is associated with vascular endothelial cell injury induced by type 2 diabetes mellitus. Exp. Ther. Med. 2018, 16, 3639-3645. [CrossRef] [PubMed]

34. Tosi, G.M.; Orlandini, M.; Galvagni, F. The Controversial Role of TGF- $\beta$ in Neovascular Age-Related Macular Degeneration Pathogenesis. Int. J. Mol. Sci. 2018, 19, 3363. [CrossRef]

35. Tosi, G.M.; Neri, G.; Caldi, E.; Fusco, F.; Bacci, T.; Tarantello, A.; Nuti, E.; Marigliani, D.; Baiocchi, S.; Traversi, C.; et al. TGF- $\beta$ concentrations and activity are down-regulated in the aqueous humor of patients with neovascular age-related macular degeneration. Sci. Rep. 2018, 8, 8053. [CrossRef] [PubMed]

36. Mandell, K.J.; Berglin, L.; Severson, E.A.; Edelhauser, H.F.; Parkos, C.A. Expression of JAM-A in the human corneal endothelium and retinal pigment epithelium: Localization and evidence for role in barrier function. Investig. Ophthalmol. Vis. Sci. 2007, 48, 3928-3936. [CrossRef] [PubMed]

37. Aiello, L.P.; Avery, R.L.; Arrigg, P.G.; Keyt, B.A.; Jampel, H.D.; Shah, S.T.; Pasquale, L.R.; Thieme, H.; Iwamoto, M.A.; Park, J.E. Vascular endothelial growth factor in ocular fluid of patients with diabetic retinopathy and other retinal disorders. N. Engl. J. Med. 1994, 331, 1480-1487. [CrossRef]

38. Giocanti-Aurégan, A.; Hrarat, L.; Qu, L.M.; Sarda, V.; Boubaya, M.; Levy, V.; Chaine, G.; Fajnkuchen, F. Functional and Anatomical Outcomes in Patients with Serous Retinal Detachment in Diabetic Macular Edema Treated With Ranibizumab. Investig. Ophthalmol. Vis. Sci. 2017, 58, 797-800. [CrossRef]

39. Otani, T.; Kishi, S.; Maruyama, Y. Patterns of diabetic macular edema with optical coherence tomography. Am. J. Ophthalmol. 1999, 127, 688-693. [CrossRef]

40. Shereef, H.; Comyn, O.; Sivaprasad, S.; Hykin, P.; Cheung, G.; Narendran, N.; Yang, Y.C. Differences in the topographic profiles of retinal thickening in eyes with and without serous macular detachment associated with diabetic macular oedema. Br. J. Ophthalmol. 2014, 98, 182-187. [CrossRef]

41. Ventriglia, G.; Mancarella, F.; Sebastiani, G.; Cook, D.P.; Mallone, R.; Mathieu, C.; Gysemans, C.; Dotta, F. miR-409-3p is reduced in plasma and islet immune infiltrates of NOD diabetic mice and is differentially expressed in people with type 1 diabetes. Diabetologia 2020, 63, 124-136. [CrossRef]

42. Marabita, F.; de Candia, P.; Torri, A.; Tegnér, J.; Abrignani, S.; Rossi, R.L. Normalization of circulating microRNA expression data obtained by quantitative real-time RT-PCR. Brief. Bioinform. 2016, 17, $204-212$. [CrossRef] [PubMed]

43. Vandesompele, J.; De Preter, K.; Pattyn, F.; Poppe, B.; Van Roy, N.; De Paepe, A.; Speleman, F. Accurate normalization of real-time quantitative RT-PCR data by geometric averaging of multiple internal control genes. Genome Biol. 2002, 3, RESEARCH0034. [CrossRef] [PubMed]

44. Andersen, C.L.; Jensen, J.L.; Ørntoft, T.F. Normalization of real-time quantitative reverse transcription-PCR data: A model-based variance estimation approach to identify genes suited for normalization, applied to bladder and colon cancer data sets. Cancer Res. 2004, 64, 5245-5250. [CrossRef] [PubMed] 
45. Perkins, J.R.; Dawes, J.M.; McMahon, S.B.; Bennett, D.L.H.; Orengo, C.; Kohl, M. ReadqPCR and NormqPCR: $\mathrm{R}$ packages for the reading, quality checking and normalisation of RT-qPCR quantification cycle (Cq) data. BMC Genom. 2012, 13, 296. [CrossRef]

46. Grieco, G.E.; Cataldo, D.; Ceccarelli, E.; Nigi, L.; Catalano, G.; Brusco, N.; Mancarella, F.; Ventriglia, G.; Fondelli, C.; Guarino, E.; et al. Serum Levels of miR-148a and miR-21-5p Are Increased in Type 1 Diabetic Patients and Correlated with Markers of Bone Strength and Metabolism. Non-Coding RNA 2018, 4. [CrossRef]

(C) 2020 by the authors. Licensee MDPI, Basel, Switzerland. This article is an open access article distributed under the terms and conditions of the Creative Commons Attribution (CC BY) license (http://creativecommons.org/licenses/by/4.0/). 